\title{
\%
}

\section{EL PLAN DE ACCIÓN PARA LA INTERNACIONALIZACIÓN DE LA ECONOMÍA ESPAÑOLA 2021-2022}

El Consejo de Ministros aprobó, el 11 de mayo de 2021, el Plan de Acción para la Internacionalización de la Economía Española 2021-2022, tercer plan bienal que desarrolla la Estrategia de Internacionalización de la Economía Española 2017-2027. Este plan ha sido elaborado por el Ministerio de Industria, Comercio y Turismo a través de la Secretaría de Estado de Comercio y sus organismos e instrumentos dependientes, con la participación de todos los ministerios con actividad en materia de internacionalización, consultadas las comunidades autónomas y el sector privado. Tiene como objetivo impulsar la internacionalización de la economía española y posicionar al sector exterior como pilar de la recuperación económica y generador de empleo, con carácter estructural, a través de la mejora de la competitividad y la mayor presencia de las empresas españolas en el exterior. Sus medidas siguen las líneas orientativas que señaló el Plan de Choque frente a la COVID-19, puesto en marcha desde el inicio de la pandemia para mitigar el impacto de la crisis sobre el sector exterior y proteger el tejido exportador hasta que pueda recobrar su actividad con normalidad. Se busca que los instrumentos y herramientas de apoyo a la internacionalización ayuden a la transformación del sector exterior y a su adaptación a las nuevas tendencias, siendo más competitivo y resiliente. Asimismo, estas orientaciones están alineadas con el Plan de Recuperación, Transformación y Resiliencia.

Palabras clave: sector exterior, internacionalización, política comercial.

Clasificación JEL: F13, F14, F20.

\section{Introducción}

La internacionalización de la economía española debería ocupar un relevante papel en la recuperación económica en la era pospandemia,

* Secretaría de Estado de Comercio. Este artículo ha sido elaborado por Carmen Laín Valenzuela, Subdirectora General de Estrategia de Internacionalización, y por Pablo González Llorente, Jefe de Servicio de la Subdirección General de Estrategia de Internacionalización.

Versión de septiembre de 2021.

DOI:https://doi.org/10.32796/bice.2021.3138.7283 a través de la reactivación del comercio y de la inversión directa, como factores dinamizadores. Antes de la llegada de la COVID-19, la economía española presentaba un crecimiento económico sólido y sostenido, así como un superávit por cuenta corriente continuado. La irrupción de la pandemia, que exigió poner en pausa largos sectores de la economía y, notablemente, el sector exterior, provocó un fuerte shock económico, causando una grave recesión mundial en 2020, mucho más profunda que la de 2009. $\triangleright$ 
Se trata de una crisis global, pero su impacto está siendo distinto en función de la evolución epidemiológica y de la estructura económica de cada país. En España ha provocado una caída del PIB del 10,8\% en el año 2020.

Consecuentemente, se hizo necesario adaptar los instrumentos y organismos de apoyo a la internacionalización e implementar medidas inmediatas, con especial atención a las pymes, para mitigar el impacto de la crisis sobre el sector exterior y proteger el tejido exportador. Desde que se inició la pandemia, la Secretaría de Estado de Comercio y sus organismos e instrumentos dependientes (ICEX, CESCE, COFIDES, FIEX/FONPYME y FIEM) han estado muy cerca de las empresas exportadoras y han logrado adaptar en poco tiempo una gran parte de sus instrumentos al nuevo contexto. El conjunto de medidas del Plan de Choque frente a la COVID-19 en apoyo a la internacionalización, puesto en marcha por la Secretaría de Estado de Comercio, ha movilizado 2.643 millones de euros. Además de medidas inmediatas para proteger al sector exportador, también recoge las actuaciones destinadas a promover la imagen de España asociada a la competitividad y a la excelencia productiva, y las realizadas ante la UE y en foros multilaterales como la OMC, la OCDE o el G20 para mantener los mercados abiertos ante los crecientes riesgos de proteccionismo y preferencia por el consumo nacional.

Manteniendo las actuaciones del Plan de Choque frente a la COVID-19 mientras sea necesario, las medidas del Plan de Acción para la Internacionalización de la Economía Española 2021-2022, objeto de este artículo, se han elaborado conforme a las líneas orientativas que señaló dicho plan, para que los instrumentos y herramientas de apoyo a la internacionalización de la economía española ayuden a la transformación del sector exterior y que se pueda adaptar a las nuevas tendencias, siendo más competitivo y resiliente. En este sentido y, aunque previa a la pandemia, es de necesaria mención la Estrategia de Internacionalización de la Economía Española 2017-2027, aprobada por el Consejo de Ministros en septiembre de 2017, desarrollada por planes de acción con carácter bienal, que buscan su adaptación a las circunstancias cambiantes que condicionan la situación de nuestro sector exterior. La estrategia y los planes de acción constituyen el marco estratégico a medio-largo plazo que permite planificar las políticas del Gobierno en apoyo a la internacionalización, facilitar la coordinación de los distintos actores implicados, particularmente la colaboración público-privada, y mejorar el acceso por parte de las empresas a los distintos instrumentos de apoyo. El Plan de Acción para la Internacionalización de la Economía española 2021-2022 es el tercer plan bienal desde que se aprobó la estrategia.

Su objetivo es maximizar la contribución del sector exterior al crecimiento de carácter estructural, potenciando la creación de empleo de calidad y la mejora de la competitividad. Se busca apoyar a las empresas españolas en sus aspiraciones internacionales, teniendo en cuenta las dificultades que ha supuesto y todavía supone el cambio de paradigma del comercio internacional provocado por la pandemia, dotando a la base exportadora de los recursos necesarios para mantener su vocación exportadora y ser capaz de adaptarse a las nuevas circunstancias y aprovechar las nuevas oportunidades.

Asimismo, debe tenerse en consideración que la pandemia ha exacerbado ciertas tendencias proteccionistas y ha impulsado la relocalización de sectores estratégicos. Además, existen amenazas e incertidumbre asociadas al Brexit. Por consiguiente, un apoyo firme por parte de la Administración a la $\triangleright$ 
internacionalización de nuestras empresas se configura como fundamental para poder afrontar el futuro, tanto a corto como a largo plazo.

Así, el Plan de Acción 2021-2022 parte del análisis del sector exterior e identifica una serie de fortalezas y debilidades de la situación económica española en materia de internacionalización. Respecto a las fortalezas cabe destacar, entre otras, el aumento continuado de la base exportadora, contando con más de 55.000 empresas que exportan de manera regular; la mayor diversificación de las exportaciones españolas, con una menor dependencia de la zona euro; el creciente peso del comercio exterior de servicios y la inserción de España en cadenas globales de valor algo por encima del promedio de los países de la UE y la OCDE. Por otro lado, en cuanto a las debilidades, se pueden subrayar, entre otras, el reducido tamaño medio de los exportadores, asociado a una menor productividad que limita el potencial exportador; el todavía insuficiente contenido tecnológico de las exportaciones, con escaso peso de los sectores de intensidad tecnológica alta y la aún insuficiente, aunque creciente, diversificación de nuestras exportaciones, con una presencia moderada en mercados como EE. UU. y China.

Asimismo, frente a las amenazas antes mencionadas, el Plan de Acción 2021-2022 también señala oportunidades a explotar en materia de internacionalización, resaltando aquellas asociadas a la implantación de nuevas tecnologías y la digitalización de la economía, así como oportunidades de negocio asociadas a la transición ecológica a nivel internacional.

A partir de este análisis, el plan determina una serie de objetivos y medidas para fomentar la internacionalización de la economía española, que se enmarcan en los seis ejes prioritarios establecidos en la Estrategia de Internacionalización de la Economía Española 2017-2027 (Esquema 1).

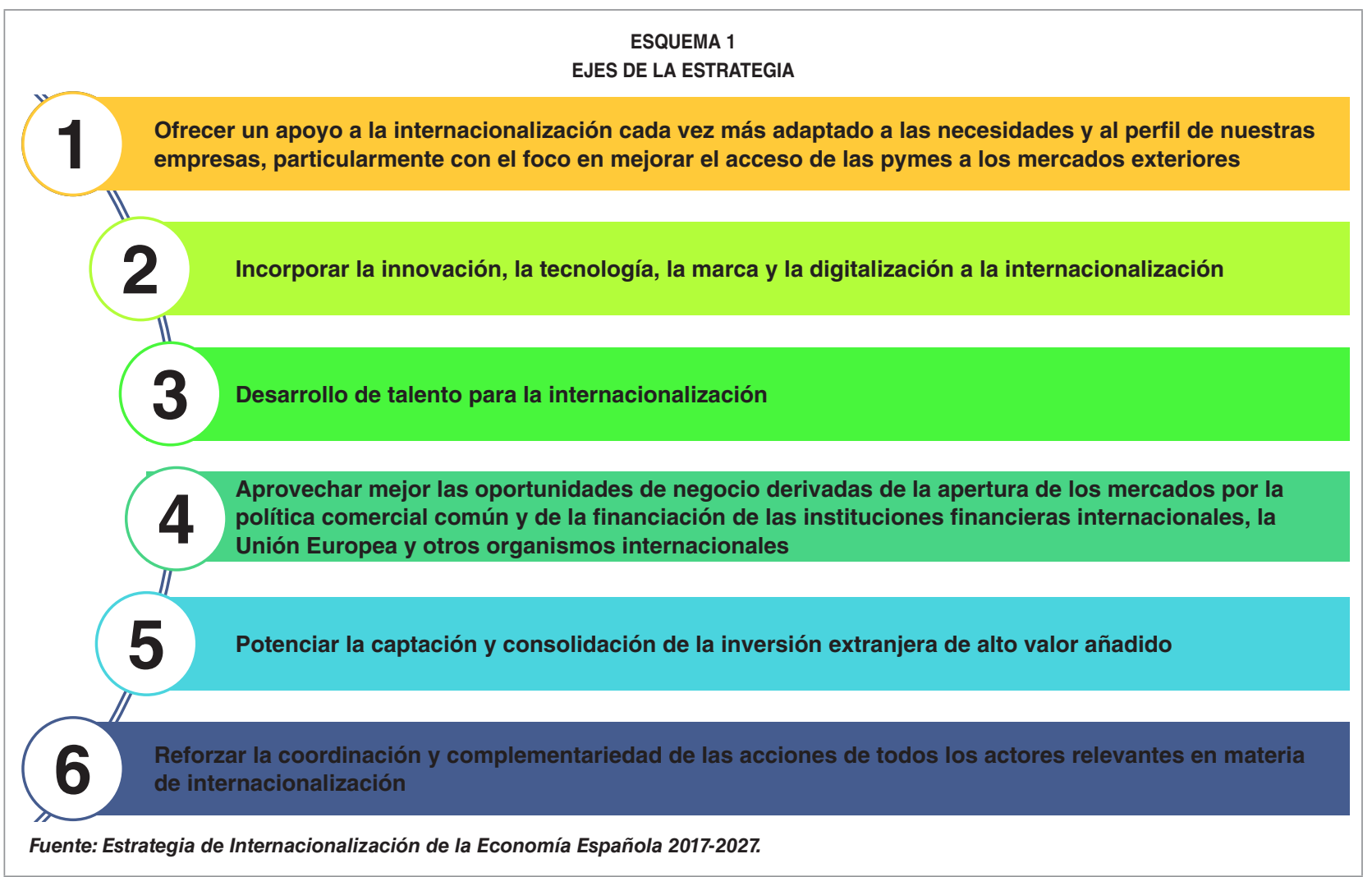


Teniendo en cuenta el análisis de fortalezas y debilidades de la internacionalización de nuestra economía, las medidas (que se analizan en el cuarto apartado de este artículo) se han elaborado conforme a las líneas orientativas señaladas en el Plan de Choque, que son las siguientes:

- Conseguir que el sector exterior se configure como pilar de crecimiento y empleo. Para ello se continuará favoreciendo la diversificación de las exportaciones y la implantación de nuestras empresas en sectores y destinos estratégicos, con atención a las oportunidades que puedan derivarse de la crisis.

Las acciones del plan se concentran en los sectores de hábitat, moda, cadena de valor agroalimentaria, sector salud, sector de tecnologías de la información y comunicaciones, infraestructuras (incluidas energías renovables, movilidad y agua), sector del automóvil e industrias culturales, con especial atención al sector audiovisual. En todos los sectores se prestará especial atención a asegurar la sostenibilidad e incorporar la transición ecológica, la digitalización y la tecnología. En colaboración con la Secretaría de Estado de Turismo, y dado el fuerte impacto de la pandemia sobre el sector turístico español, líder mundial y con un elevado peso en el PIB y el empleo de nuestro país, este plan de acción pondrá el foco en la internacionalización de la empresa turística española, especialmente las de tamaño medio y pequeño altamente especializadas, con modelos de negocio singulares.

Se buscará atraer la inversión extranjera directa en sectores estratégicos, asegurando la coherencia con la política industrial y los planes sectoriales, incluyendo los planes ambientales y de lucha contra el cambio climático, así como las oportunidades que surjan en la reorganización de las cadenas globales de valor. Se reforzará el papel de ICEX-Invest in Spain, clave en la atracción de inversión extranjera directa y detección de barreras. Los sectores de atención preferente serán las industrias de alto contenido tecnológico que contribuyan a los objetivos de la doble transición ecológica y digital, haciendo especial hincapié en los sectores y subsectores relacionados con la movilidad eléctrica y nuevas formas limpias de propulsión, las energías renovables, la optimización de la producción agroalimentaria y ciencias de la vida.

Se prevé la revisión de la Estrategia PASE (Países con Actuación Sectorial Estratégica), con la incorporación de Reino Unido tras el Brexit.

- Aumentar la resiliencia de nuestro sector exterior. Potenciar la capacidad de resiliencia de nuestro sector exterior con instrumentos suficientes y adecuados a sus necesidades, con especial atención a las pymes. Todos los instrumentos públicos al alcance de las empresas están siendo revisados para conseguir que nuestras empresas exportadoras sean más fuertes.

Acompañar a las empresas internacionalizadas en el cambio estructural necesario (y acelerado por las circunstancias) hacia la digitalización y el uso masivo de tecnologías.

Refuerzo de la seguridad en las cadenas globales de valor en las que $\triangleright$ 
España está integrada, mediante la creación de un marco estable, basado en reglas, diversificando las fuentes de oferta, abriendo nuevos mercados y desarrollando marcos cooperativos para un acceso a productos críticos, en línea con lo establecido en la Comunicación de la Comisión Europea sobre la Revisión de la Política Comercial de febrero de 2021.

Consolidar la sostenibilidad como herramienta de internacionalización en una estrategia empresarial vinculada a potenciar el impacto social y medioambiental en los mercados de destino, a reducir el riesgo ante marcos regulatorios potencialmente inciertos o cambiantes y como elemento dinamizador de la actividad de las empresas españolas en el exterior.

Por otro lado, la Estrategia de Internacionalización 2017-2027 incorpora un ejercicio de evaluación que permite, entre otros aspectos, medir los resultados y el nivel de cumplimiento de las metas fijadas, así como valorar el grado de eficacia y de eficiencia de los planes bienales y las actuaciones y medidas asociadas a los mismos. La evaluación del Plan de Acción 2017-2018 ya ha sido publicada, mientras que la correspondiente al Plan de Acción 20192020 está prevista para el segundo semestre de 2021. Por último, se espera evaluar el Plan de Acción 2021-2022 en el año 2023.

Finalmente, conviene señalar que el Plan de Acción 2021-2022 se trata de un plan integrador, basado en los principios de coherencia y coordinación en la actuación del Estado y de complementariedad con el sector privado, en el que han participado los organismos de apoyo a la internacionalización, tanto comerciales como financieros (ICEX España Exportación e Inversiones, CESCE, COFIDES e ICO), así como los distintos ministerios que contribuyen con sus políticas a la internacionalización, coordinados por el Ministerio de Industria, Comercio y Turismo. Además, se han tenido muy en cuenta las orientaciones proporcionadas por el sector privado, en particular la Cámara de España y CEOE, y han sido consultadas las comunidades autónomas. Este plan es, por tanto, el resultado de un esfuerzo notable de coordinación y cooperación entre todos los actores implicados en la internacionalización de nuestra economía.

\section{Situación del sector exterior español}

A pesar de las incertidumbres que aún subsisten, como las asociadas a la propia evolución de la pandemia, el Brexit, el cambio climático o el incremento de las medidas proteccionistas, las perspectivas de recuperación de la actividad económica y comercial internacional para 2021 son favorables, tras el fuerte impacto de la crisis sanitaria en 2020 . Estas previsiones pueden estar reflejando una mejor adaptación de las economías a las condiciones de la pandemia, el despliegue de las campañas de vacunación y la adopción de paquetes de medidas de reactivación en las principales economías, y configuran un marco más favorable a la recuperación del sector exterior español. Esta mejora del escenario se produce tras un año 2020 en el que algunos de los sectores, como los vinculados a las actividades de servicios que requieren movimientos internacionales de personas (en especial el turismo), o los relacionados con bienes de inversión o de consumo duradero, acusaron con intensidad los efectos de la crisis sanitaria. 
Cabe señalar varias características positivas del sector exterior español, consecuencia de su tendencia y evolución reciente, que pueden contribuir al aprovechamiento del escenario más favorable que se abre en 2021. Estas son, principalmente, el hecho de contar con una economía cada vez más abierta al exterior, el aumento de la base exportadora y una mayor diversificación de las exportaciones.

El grado de apertura de la economía española, calculado como la suma de exportaciones e importaciones de bienes y servicios respecto al PIB, ha aumentado de forma robusta durante la última década, lo que se demuestra por los casi 20 puntos de incremento presentados en el año 2019 en comparación con los datos de 2009, situándose en el $66,9 \%$. El descenso de este indicador en 2020 tiene un carácter marcadamente coyuntural, vinculado al impacto de la pandemia. También, estos datos ilustran el gran peso que supone el sector exterior para nuestra economía, de forma similar a lo que ocurre en países de nuestro entorno, como Alemania, Francia, Italia o Reino
Unido. En el Gráfico 1 se observa la evolución de los respectivos grados de apertura de dichos países.

En cuanto a la evolución de la base exportadora, el Gráfico 2 muestra cómo, tras un continuado incremento, se ha llegado a contar en 2020 con más de 55.000 exportadores que lo hacen de forma regular, lo que revela una consolidación de la posición de estas empresas en los mercados internacionales. Se trata de empresas que han realizado operaciones de exportación, al menos durante cada uno de los últimos cuatro años, lo que indica que la exportación ha pasado a ser parte integrante de su operativa habitual y no un recurso transitorio ante una situación de debilidad de la demanda interna. Este crecimiento de la base de exportadores regulares constituye un excelente punto de partida para aprovechar las oportunidades que puedan ofrecer los mercados internacionales en los próximos años.

Respecto a la diversificación de las exportaciones, esta se ha visto reforzada durante los últimos veinte años, reduciéndose la $\triangleright$

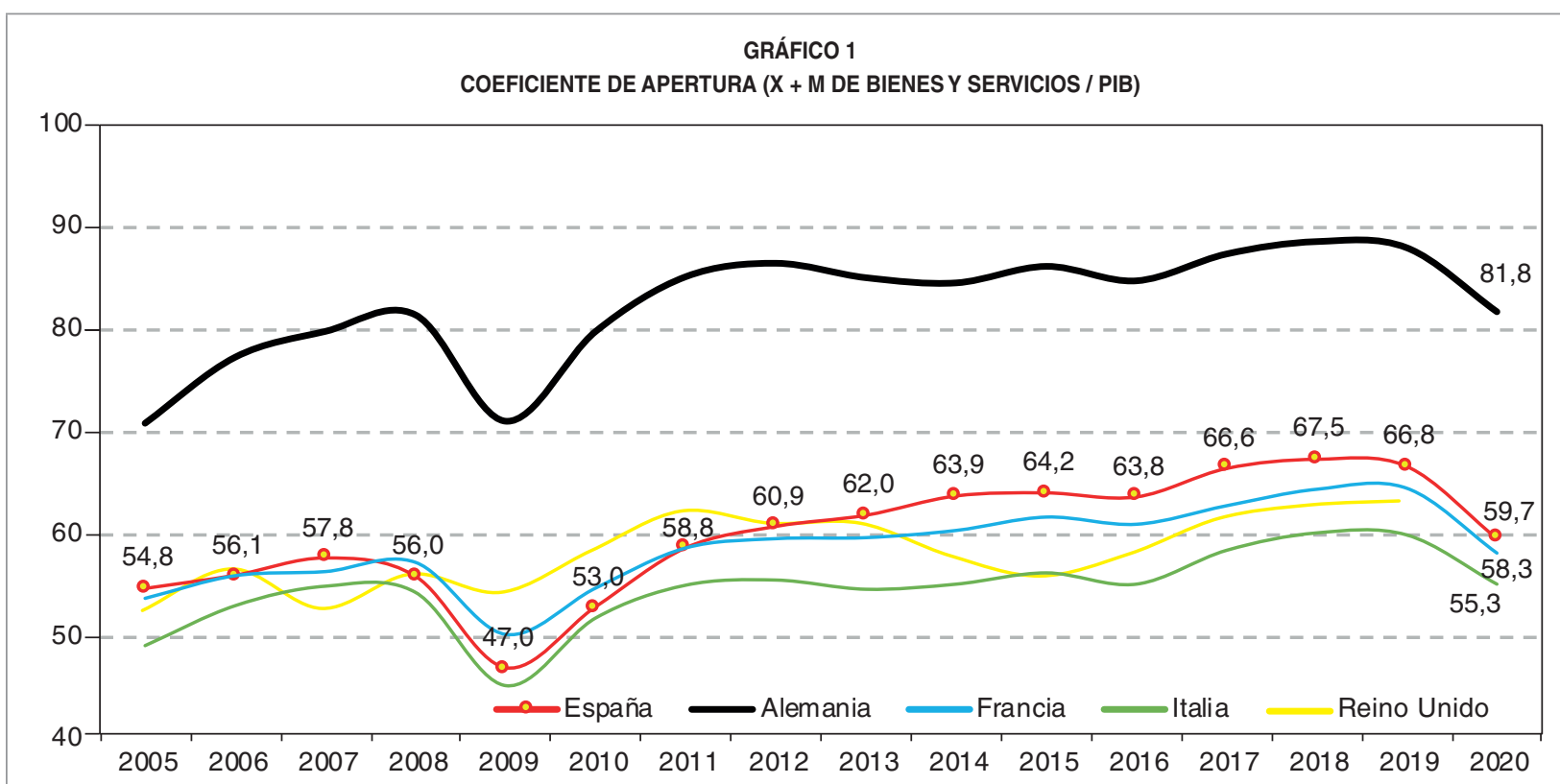

Fuente: Eurostat, National Accounts. Actualizado: 12/04/21. 


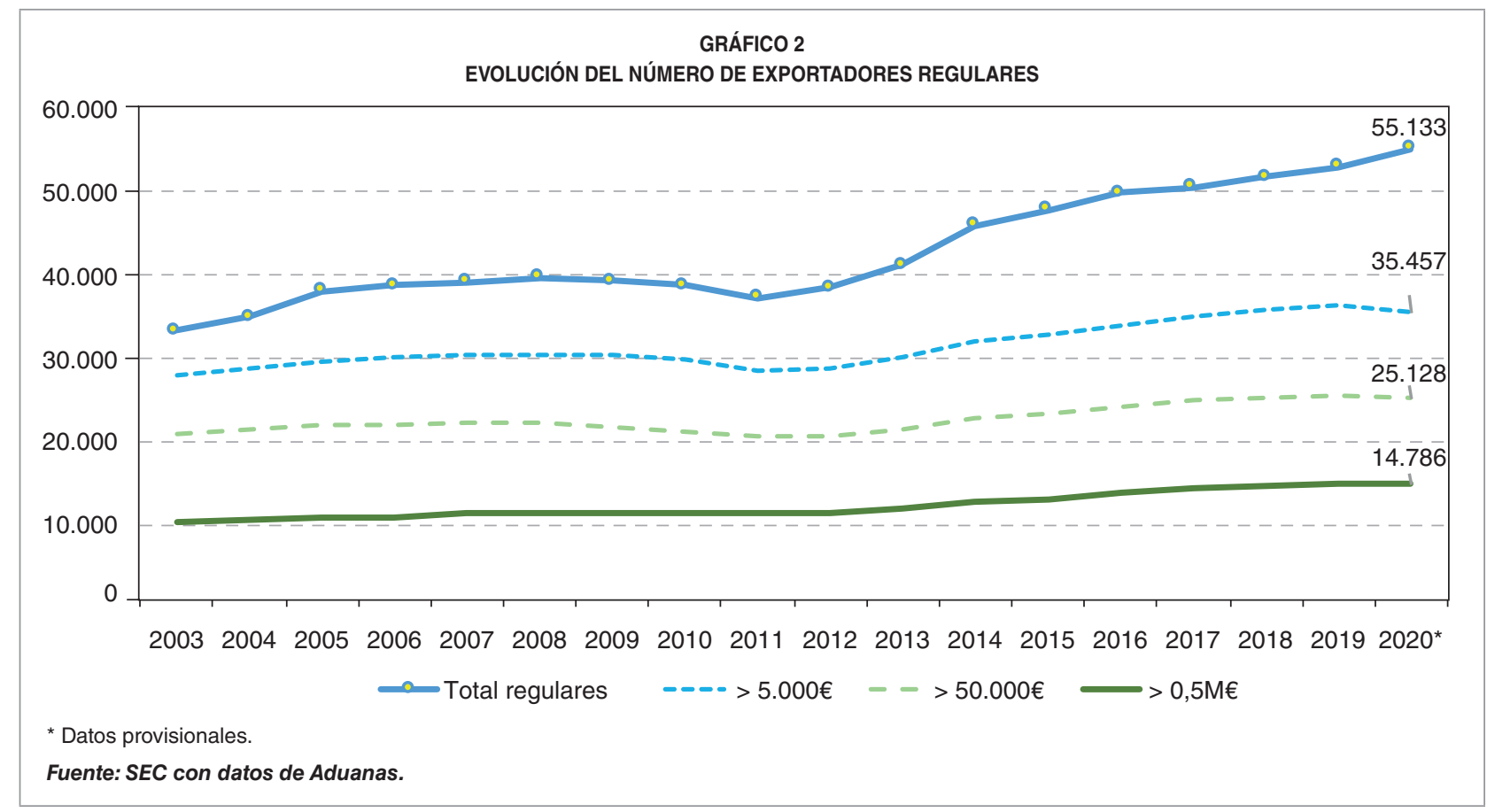

dependencia respecto a la Unión Europea, tal y como se observa en el Gráfico 3. No obstante, aún existe un margen de mejora considerable en ciertos mercados con un gran peso internacional, como Estados Unidos, India o China.

También cabe hacer referencia a la fuerte tendencia positiva presentada en el comercio exterior de servicios, y más específicamente en los servicios no turísticos, al haberse cuadruplicado sus exportaciones en 2019 respecto a los datos del año 2000.

Por último, puede destacarse un grado de inserción de España en las cadenas globales de valor algo superior al de la media de $\square$

GRÁFICO 3

DIVERSIFICACIÓN GEOGRÁFICA DE LAS EXPORTACIONES ESPAÑOLAS DE BIENES
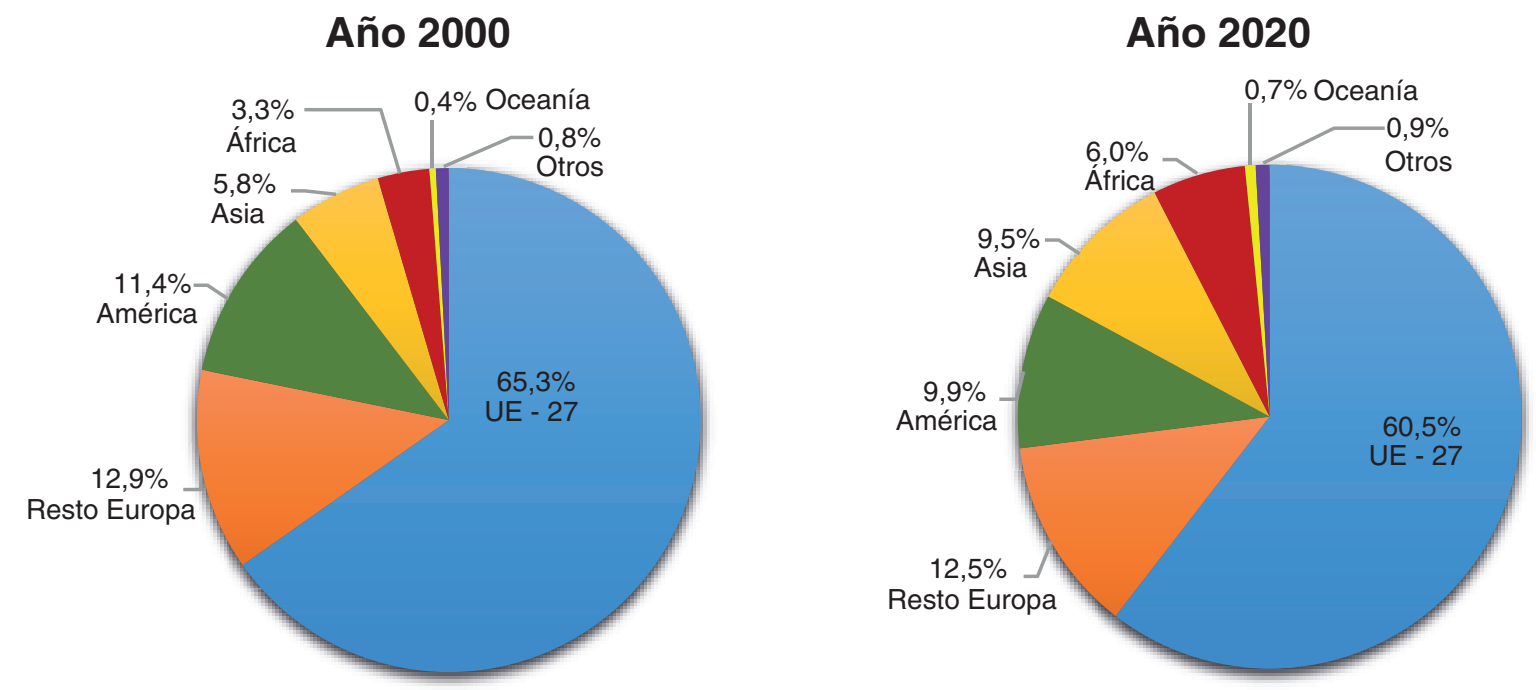

Fuente: S. G. Estudios y Evaluación de Instrumentos de Política Comercial, con datos de Aduanas. 
países de la UE y de la OCDE y muy relacionado tanto con el comercio exterior como con la inversión extranjera directa. Este dato revela la capacidad que han mostrado las empresas españolas de adaptación ante los cambios que ha registrado la organización mundial de la producción en los últimos años. Las perspectivas para los próximos años apuntan a nuevos cambios en este ámbito, en busca de una mayor resiliencia de las cadenas globales de valor, lo que puede dar lugar a oportunidades que puedan aprovechar las empresas españolas.

\subsection{Inversiones exteriores}

Como consecuencia de la crisis económica y de los altos niveles de incertidumbre asociados a la pandemia, los flujos de inversión han disminuido significativamente. Sin embargo, la inversión internacional se vuelve ahora más necesaria que nunca, tanto por la financiación que representa como por la capacidad de crear empleo y aumentar la producción que supone.

A continuación se elabora un breve análisis sobre la posición española en cuanto a la inversión internacional, tanto desde un punto de vista emisor como receptor.

\subsubsection{Inversión española en el exterior}

España puede considerarse como un inversor de referencia a nivel internacional, aunque, eso sí, su presencia es considerablemente menor que la de los países inversores líderes, como, por ejemplo, Estados Unidos o China. La inversión emitida permite acelerar la internacionalización de nuestra economía, así como alcanzar ciertos mercados que no podrían ser accesibles de otra forma. Asimismo, esta suele acarrear un aumento de las exportaciones, gracias al mejor encaje que puede suponer para el país en las cadenas globales de valor.

Respecto a los flujos de inversión bruta emitidos por España (descontadas las cifras de inversión en entidades de tenencia de valores extranjeros -ETVE-), el importe total para el año 2020 alcanzó los 23.070 millones de euros, lo que supone una variación interanual positiva del 2,2\%. En cuanto a la distribución geográfica, los países que recibieron un mayor porcentaje de inversión española fueron Luxemburgo (21,8\%), Estados Unidos (16,1\%) y Reino Unido (8,4\%). Respecto a la distribución sectorial destacan los siguientes sectores: seguros, reaseguros y fondos de pensiones $(16,4 \%)$, suministro de energía eléctrica, gas, vapor y aire acondicionado $(14,1 \%)$ y almacenamiento y actividades anexas al transporte (10,6\%). Por otra parte, la inversión neta emitida ha alcanzado en 2020 la cifra de 19.817 millones de euros (también descontando la inversión asociada a ETVE).

Se produce, como resultado, una situación en la que la inversión bruta de 2020 se ha visto disminuida en comparación con la media del periodo $2016-2020$, en un $28,7 \%$, mientras que la inversión neta ha aumentado un $28,9 \%$, también en comparación con la media del mismo periodo.

Finalmente, cabe señalar que, en términos de stock, la posición inversora total española en el exterior en 2019 alcanzó el valor de 476.551 millones de euros (excluyendo la inversión asociada a ETVE), lo que supone un incremento interanual del 4,8\%.

\subsubsection{Inversión extranjera en España}

La estrategia de atracción de inversiones exteriores, impulsada principalmente por $\triangleright$ 
ICEX- Invest in Spain, prioriza ciertos sectores, en línea con el objetivo de transformar el tejido productivo español hacia la incorporación de un mayor contenido tecnológico y buscando el cumplimiento de los Objetivos de Desarrollo Sostenible. La inversión extranjera directa (IED) es especialmente deseada puesto que impacta positivamente en la economía española gracias a la difusión de nuevas tecnologías y know-how, promoviendo la competencia y aumentando los niveles de capital humano de nuestros trabajadores.

Respecto a los flujos de inversión directa bruta recibida por España, en el año 2020 se alcanzó la cifra de 23.824 millones de euros (descontadas las cifras de inversión en ETVE), lo que representa una variación interanual negativa del $-0,8 \%$. Los países inversores de origen último con mayor protagonismo han sido Suiza (16,3\%), Estados Unidos (16,2\%) y Reino Unido (12,6\%). Respecto a la distribución sectorial cabe resaltar como principales sectores receptores de inversión los siguientes: actividades auxiliares a los servicios financieros y a los seguros (12,7\%), telecomunicaciones $(9,3 \%)$ y servicios financieros excepto seguros y fondos de pensiones (7,5\%). Por otro lado, la inversión neta recibida ha alcanzado en 2020 la cifra de 12.884 millones de euros (también descontando la inversión asociada a ETVE).

Estos valores alcanzados en 2020, tal y como ocurre en términos mundiales, representan un nivel sustancialmente inferior al presentado de media durante los últimos años. Concretamente, en 2020, la inversión bruta española fue un $24,6 \%$ inferior, mientras que la neta lo fue en un $43 \%$, en comparación con los valores medios de los flujos recibidos durante el periodo 2016-2020.

Por último, en cuanto al stock de inversión recibida, este alcanzó en 2019 (excluyendo las inversiones de ETVE) la cantidad de 481.706 millones de euros, un 2,7\% superior interanualmente.

\section{Objetivos del Plan de Acción 2021-2022}

\subsection{Objetivo general}

Tanto la Estrategia de Internacionalización de la Economía Española 2017-2027 como los planes bienales que la desarrollan persiguen un mismo objetivo general, que no es otro que impulsar la internacionalización de la economía española, consiguiendo que el sector exterior se configure, de nuevo, como pilar de la reactivación económica y del crecimiento generador de empleo, con carácter estructural, a través de la mejora en la competitividad y de la mayor presencia de nuestras empresas en el exterior.

\subsection{Objetivos específicos y objetivos intermedios}

De cara a alcanzar el objetivo general, se establecen una serie de objetivos específicos a los que se asocian unos indicadores que permiten cuantificar su grado de consecución, para realizar un seguimiento y valoración del plan, con el fin último de impulsar las mejoras que puedan resultar necesarias. Estos objetivos específicos son los siguientes:

- Aumentar la base de empresas que exportan regularmente, particularmente con el foco en mejorar el acceso de las pymes a los mercados exteriores.

- Promover la implantación de nuestras empresas en sectores estratégicos en determinados mercados. 
- Incrementar el valor añadido de las exportaciones y la inserción de nuestras exportaciones en las cadenas globales de valor.

- Aumentar la atracción de inversión extranjera directa.

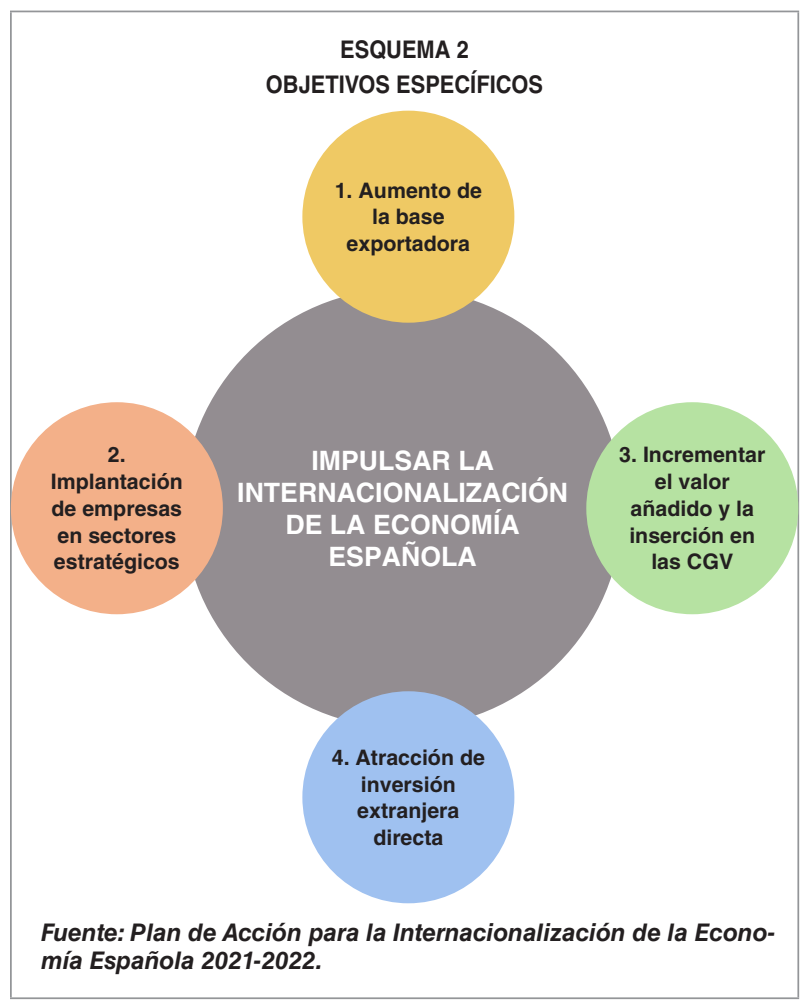

Para alcanzar los objetivos específicos se define una serie de objetivos intermedios, incidiendo cada uno de estos últimos en varios de los primeros. No obstante, las políticas necesarias para incidir en algunos de estos objetivos escapan del ámbito de la internacionalización. Por ello, solo se asocian indicadores a aquellos objetivos intermedios relacionados con las políticas de internacionalización que se llevan a cabo mediante los distintos instrumentos comerciales y financieros de apoyo a la internacionalización.

A modo de ejemplo, algunos de los objetivos intermedios incluidos en el Plan de Acción 2021-2022 son: incentivar la participación de mujeres en la internacionalización de la economía española, incorporar la innovación tecnológica, potenciar la formación en internacionalización utilizando las nuevas tecnologías o captar activamente proyectos de inversión extranjera en países y sectores prioritarios.

\section{Principales medidas del Plan de Acción 2021-2022}

Las medidas incluidas en el Plan de Acción 2021-2022 buscan alcanzar los objetivos señalados anteriormente, identificando los instrumentos y organismos encargados de su ejecución. Se enmarcan en los seis ejes definidos en la Estrategia de Internacionalización de la Economía Española 2017-2027:

Eje 1. Ofrecer un apoyo a la internacionalización cada vez más adaptado a las necesidades y al perfil de nuestras empresas, particularmente con el foco en mejorar el acceso de las pymes a los mercados exteriores

En línea con el anterior Plan de Acción 2019-2020, se deben seguir enfocando los esfuerzos en conocer mejor la realidad de las empresas exportadoras españolas, con el ánimo de poder ofrecer un apoyo adaptado a sus necesidades, alcanzando un mayor nivel de eficacia y eficiencia en la aplicación de los instrumentos utilizados, particularmente en lo respectivo a los programas y servicios de promoción de exportaciones, inversiones y de los instrumentos financieros públicos de apoyo a la internacionalización.

Se utilizarán nuevos instrumentos para conocer mejor a las empresas, como el servicio ADA para el análisis y autodiagnóstico de las capacidades de la empresa, la plataforma $\triangle$ 
CESNET y la nueva web de CESCE, así como la consolidación de la herramienta de gestión de clientes CRM en los distintos organismos de la Secretaría de Estado de Comercio.

Además, se refuerza la Red Exterior de Oficinas Económicas y Comerciales de la Secretaría de Estado de Comercio, que constituye un instrumento fundamental al realizar, entre otras, tareas de diplomacia comercial y gestiones en destino por cuenta de nuestras empresas, identificando oportunidades, elaborando información de valor y generando inteligencia comercial. Debido a su cercanía a mercados extranjeros, a sus tareas habituales se han ido añadiendo otras, cuya importancia se ha visto aumentada por la pandemia, como la identificación de suministradores críticos, en un marco donde el impacto de la pandemia en el comercio internacional ha demostrado la necesidad de reforzar la resiliencia de las cadenas de valor; las tareas de agencia y apoyo al cierre de operaciones comerciales y de inversión; la selección de socios locales y alianzas para reforzar el anclaje de las empresas en los mercados de destino con mayor presencia local; el análisis de la normativa y apoyo al compliance local, especialmente en un periodo de incremento del rol del sector público (como inversor y regulador) en la actividad económica; el refuerzo de la comunicación y de las estrategias de imagen y la generación de nuevos proyectos de inversión en España.

Por otro lado, la Red de Direcciones Territoriales y Provinciales de Comercio también ha visto resaltada su relevancia en este plan de acción, debido principalmente a su proximidad a las empresas exportadoras, lo que permite un mejor conocimiento de su situación y, a su vez, una mejor adaptación de los instrumentos utilizados para apoyarlas.
Por su parte, ICEX ofrecerá a las empresas una serie de instrumentos de apoyo a la internacionalización en función de su tipología y de sus necesidades específicas. Además, en un contexto de movilidad reducida en el que las herramientas digitales se han vuelto imprescindibles, ICEX está potenciando ya todos sus servicios digitales, aprovechando el impulso y desarrollo creciente de las plataformas de negocio digital, así como la evolución ascendente del comercio electrónico, acelerada por la pandemia. En este contexto, ICEX también ayudará a las empresas a adaptarse a la transformación digital que está marcando ya el futuro de la industria de promoción comercial.

Por otro lado, algunos nuevos programas, como ICEX Localiza, van dirigidos a apoyar la presencia local de las empresas españolas en los mercados objetivo, configurándose como una forma de reforzar la resiliencia de nuestro ecosistema empresarial.

En lo relativo a los instrumentos financieros, la competencia internacional en materia financiera obliga a una aproximación flexible y adaptada a las necesidades de las empresas para optimizar sus oportunidades de negocio. En este sentido, tanto CESCE, como FIEM, FIEX y FONPYME, ofrecen opciones de financiación a distintos tipos de proyectos. Además, se amplían las líneas FIEM/PYME y ECOFIEM, las líneas CESCE COVID-19 y las pólizas verdes; destacan también el mantenimiento del mecanismo fast-track de COFIDES y la nueva actividad financiera internacional de ICO, en particular a través del Canal Internacional.

Desde la Secretaría de Estado de Comercio, a través de sus organismos y siempre en estrecha colaboración público-privada, se continúa trabajando en acciones vinculadas con el Grupo de Trabajo sobre «Mujer e Internacionalización» con el fin de poner en marcha $\triangleright$ 
medidas concretas que mejoren el papel de las mujeres en la internacionalización de la economía española. Además, también se llevan a cabo esfuerzos a nivel internacional, siendo España signataria de la Declaración de Buenos Aires (2017) y, en el europeo, apoyando firmemente la inclusión de un capítulo sobre género en todos los acuerdos comerciales que negocie la UE.

Por último, se considera fundamental reforzar las medidas e instrumentos destinados a proteger e impulsar la sostenibilidad, todo ello en línea con la Agenda 2030 y los Objetivos de Desarrollo Sostenible, otorgando un papel protagonista al sector privado, y más concretamente a las empresas exportadoras españolas.

Eje 2. Incorporar la innovación, la tecnología, la marca y la digitalización a la internacionalización

La innovación, la tecnología, la marca y la digitalización se configuran como fundamentales en la internacionalización de la economía española. Esto se puede ilustrar por la mayor calidad y competitividad que permite un nivel superior de innovación en nuestras empresas, habiendo aumentado la especialización exportadora española en productos de tecnología de media y alta gama, representando hoy más del $50 \%$ de las ventas al exterior. Además, las pymes innovadoras tienen un elevado peso en la financiación de la I+D, acometiendo casi el $50 \%$ de la I+D total, frente al $7 \%$ en Japón, el $11 \%$ en Alemania o el $17 \%$ en EE. UU.

Respecto a las marcas, estas permiten una diferenciación de nuestras empresas y sus productos, aumentando también su nivel de competitividad. Las marcas se han convertido en motores del crecimiento y la competitividad, generando además un efecto determinante sobre la propia imagen comercial del país de origen. Destacan la campaña de ICEX con el Ministerio de Agricultura "Spain Food Nation" y la campaña de ICEX con Cámara de España y Foro de Marcas Renombradas «Think Again, Think Spain».

La digitalización, a su vez, constituye uno de los principales motores transformadores de la economía presente y futura, siendo tanto fuente de oportunidades como una necesidad, puesto que la pandemia ha enfatizado la necesidad de que los sectores tradicionales también han de adaptarse y reinventarse. Consecuentemente, las empresas españolas deben esforzarse por digitalizarse en su proceso de internacionalización, ya que su éxito depende, en gran medida, de conseguir aprovechar las oportunidades relacionadas con la transición tecnológica y digital.

\section{Eje 3. Desarrollo de talento para la} internacionalización

La presencia de talento para la internacionalización es una cuestión absolutamente clave para que aquella se pueda realizar satisfactoriamente, es decir, es condición necesaria para la competitividad internacional de nuestras empresas. Su importancia, además, se observa fácilmente, dado que se estima que más de tres millones de puestos de trabajo en España dependen de la demanda externa. Además, existen 10.135 filiales de empresas españolas en el exterior que emplean a 1.668 .706 personas.

Por consiguiente, la Administración está creando y ofreciendo una amplia oferta formativa, de másteres, cursos y programas a través de nuevos formatos no presenciales, asíncronos, y con tendencia al microaprendizaje y el seguimiento adaptativo del alumno a través de inteligencia artificial. 
Algunos de los campos donde se está haciendo mayor hincapié para la formación en la internacionalización son: el transporte y la logística, la digitalización y la sostenibilidad medioambiental y social. Desde la perspectiva de la política industrial se impulsará la colaboración público-privada para el desarrollo de programas formativos en disciplinas STEM (ciencia, tecnología, ingeniería y matemáticas, por sus siglas en inglés) que aúnen formación y prácticas o trabajo en las empresas patrocinadoras.

Por otra parte, se debe mencionar la labor de las universidades, centros de formación profesional e ICEX-CECO, determinantes en el desarrollo de talento para la internacionalización. No obstante, todavía se considera que hay una insuficiente oferta de prácticas laborales relacionadas con el comercio internacional, de gran utilidad para potenciar las habilidades de comunicación, de negociación y de trabajo en equipo. Por esta razón, el Plan de Acción 2021-2022 prevé un impulso de los programas de becas y de las prácticas en empresas internacionalizadas.

También se mantendrán y potenciarán los programas de movilidad laboral internacional, para apoyar la atracción de talento extranjero, facilitando la entrada de profesionales altamente cualificados, investigadores e inversores. En este sentido, destaca el portal «Empléate».

Eje 4. Aprovechar mejor las oportunidades de negocio derivadas de la apertura de los mercados por la política comercial común y de la financiación de las instituciones financieras internacionales, la Unión Europea $y$ otros organismos internacionales

Con la llegada de la COVID-19 se ha puesto de manifiesto la gran relevancia de asegurar los suministros y proteger las cadenas de valor, para lo cual la política comercial y el marco institucional internacional es fundamental.

Como resultado, defender un sistema multilateral, integrador, transparente y justo se ha convertido en una necesidad. Sin embargo, las acciones dirigidas a tal objetivo deben ser complementadas por medidas dirigidas a promover la resiliencia, diversificando los suministradores.

Por consiguiente, España debe continuar contribuyendo activamente en el ejercicio iniciado por la Comisión para la revisión de la política comercial de la UE, con la visión de lograr una «Autonomía Estratégica Abierta» para Europa y centrada en el fortalecimiento de la capacidad de la UE para defender los intereses de sus operadores económicos en los planos multilateral, bilateral e interno. La iniciativa tiene por objetivo responder a los nuevos retos mundiales, como la digitalización, el cambio climático, la sostenibilidad y la proliferación de medidas proteccionistas. También lograr una relación económica más equilibrada con nuestros socios comerciales.

Por otra parte, la pandemia también ha puesto de manifiesto la necesidad de reforzar la cooperación y la gobernanza internacional, a través de la OMC, la OCDE, el G20 y las instituciones financieras internacionales. Por tanto, se debe continuar apoyando el sistema multilateral, al mismo tiempo que se debe reforzar la situación de España en dicho ámbito para, entre otros fines, coadyuvar a incrementar el éxito de las empresas españolas en las licitaciones internacionales. A través de la Red Exterior de Oficinas Económicas y Comerciales de la Secretaría de Estado de Comercio se fomentará la participación de empresas españolas en licitaciones internacionales, reforzando sus herramientas de inteligencia empresarial para profundizar en el conocimiento de las licitaciones internacionales y poder mejorar el posicionamiento de España en los procesos de adjudicación. 
Además, el plan busca apoyar a nuestras empresas ante el Brexit, con instrumentos como las «Guías de acceso al mercado británico», el Canal Brexit como ventana para el diálogo entre empresa y Administración, y un descuento en el precio de los servicios personalizados de ICEX (cheque Brexit).

\section{Eje 5. Potenciar la captación y consolidación} de la inversión extranjera de alto valor añadido

Tal y como se ha expuesto más arriba, como resultado de la incertidumbre asociada a la pandemia, los flujos de inversión cayeron abruptamente en 2020, tanto a nivel mundial como nacional. De acuerdo con los datos disponibles de la UNCTAD, en el primer semestre del año 2020, la IED a nivel mundial cayó en un $49 \%$. En España, los flujos de inversión también se vieron afectados, pero la caída no fue tan pronunciada como en otras economías.

Así, se ha producido una situación en la que la gran caída de los flujos de IED se une a la gran importancia que tiene la misma en la recuperación económica. Teniendo esto en cuenta, así como la reorganización de las cadenas globales de valor, la competencia por atraer la IED se puede exacerbar en los países de nuestro entorno. Consecuentemente, se vuelve primordial que el sector público siga trabajando por crear un entorno atractivo y favorable para los inversores, para potenciar el crecimiento sostenible, el empleo de calidad, la innovación y la atracción de talento. La seguridad jurídica, la simplificación de la regulación y la transparencia normativa se configuran como aspectos claves en esta materia. Es preciso, por tanto, conseguir un marco regulatorio cada vez más favorable para el clima de negocios y el entorno empresarial.
Asimismo, debe mencionarse el esfuerzo realizado por ICEX-Invest in Spain, mejorando el clima de negocios, potenciando su actividad de policy advocacy y reforzando su política de aftercare y de retención de la inversión. También se reforzará la estrategia de atracción de inversiones de América Latina, se apoyará la inversión en I+D+i y la atracción de talento, y se trabajará en 2021-2022 en un programa de captación de inversiones ligadas a los ejes del programa de transformación de la economía española.

En el marco del Plan de Recuperación, Transformación y Resiliencia, se pondrá en marcha el programa INNOVA INVEST, dirigido a la atracción de inversión extranjera en I+D+i y que priorizará aquellos proyectos sostenibles relacionados con la transición ecológica y la digitalización, que apoyen el crecimiento del PIB y del empleo en España.

Eje 6. Reforzar la coordinación y complementariedad de las acciones de todos los actores relevantes en materia de internacionalización

Como consecuencia de la multiplicidad de actores públicos y privados y la gran variedad de medidas e instrumentos utilizados en materia de internacionalización, el impulso de la colaboración y coordinación es una prioridad, con la meta de ahorrar costes, racionalizar recursos y lograr una mayor cohesión en la imagen-país transmitida. Las actuaciones y medidas del sector público deben regirse por el principio de coherencia, así como de complementariedad con el sector privado.

Por otro lado, muchas otras políticas influyen de forma directa o indirecta en la política de internacionalización de la economía española, debiendo ser cohesionadas entre sí, para alcanzar mejores resultados. Particularmente, $\triangleright$ 
se deben tener en cuenta, entre otras, la política industrial y otras políticas de otros ámbitos como la igualdad de género, el transporte y la movilidad internacional o la transición ecológica. Es importante subrayar que este plan se ha realizado en el marco del Grupo de Trabajo Interministerial de Apoyo a la Internacionalización, en el que participan todos los departamentos ministeriales con actividad en internacionalización y que, entre otros, han contribuido señalando sinergias entre las políticas de internacionalización y otros ámbitos de la Administración.

También destaca la importancia de potenciar la coherencia de las actuaciones de la Administración comercial española, en particular de la Red Exterior de Oficinas Económicas y Comerciales con los servicios centrales, las direcciones territoriales y provinciales de comercio, así como el papel relevante de las cámaras de comercio españolas oficiales en el exterior en apoyo a la internacionalización, siempre garantizando la complementariedad con la labor de las oficinas económicas y comerciales.

De igual modo, el plan sigue las orientaciones del sector privado, particularmente de la Cámara de Comercio de España y de la CEOE, y de las comunidades autónomas, que han colaborado en el marco del Consejo Interterritorial de Internacionalización.

\section{Prioridades geográfico-sectoriales: PASE (Países con Actuación Sectorial Estratégica), Brexit, África y América Latina}

Con el Plan de Acción 2021-2022 se prevé la revisión de la Estrategia PASE, de cara a mantener actualizadas aquellas zonas geográficas y sectores más relevantes para fomentar la exportación de productos y servicios españoles.
Con esta estrategia se busca identificar aquellos países y sectores en los que existe un mayor potencial exportador español, es decir, en los que la presencia exportadora española no es tan protagonista como debería, existiendo un amplio margen de mejora.

De cara a determinar dichos países y sectores estratégicos, en el marco del plan de acción anterior se elaboró un análisis cuantitativo y se utilizó la información proporcionada por la Red de Oficinas Económicas y Comerciales en el exterior y el ICEX, que permitió una selección inicial de países: Brasil, México, Canadá, Estados Unidos, Sudáfrica, Marruecos, Turquía, Rusia, China, Corea, India y Japón. En estos países se identificaron sectores y actuaciones prioritarias que se desarrollarían en 2019 y 2020.

En el escenario actual, teniendo en cuenta que 2020 ha sido particularmente complicado para el sector exterior, se ha decidido que la Estrategia PASE debe continuar enfocada en esos mismos países, añadiendo a Reino Unido por los efectos del Brexit. En todo caso, se podrá ampliar esta estrategia a otros mercados donde las empresas españolas aún están escasamente presentes, como el sur de Asia y África.

Por otra parte, África es para España una región de especial interés, entre otros, por motivos comerciales y económicos. Por tanto, se han incorporado al programa del Gobierno «Foco África 2023» los principios, las prioridades y las acciones identificados en el documento de política comercial y financiera para África «Horizonte África», elaborado por la Secretaría de Estado de Comercio.

Por último, el plan señala que España continuará su compromiso con la región latinoamericana en los ámbitos económico, comercial y de inversiones. 


\section{Conclusión}

El Plan de Acción para la Internacionalización de la Economía Española 2021-2022 desarrolla la Estrategia de Internacionalización de la Economía Española 2017-2027 en línea con el compromiso de reforzar la política de internacionalización de nuestro país, tanto en el corto como en el medio-largo plazo. Siendo su objetivo último el de consolidar el sector exterior como pilar estructural del crecimiento sostenible y del empleo, este plan se adapta a las circunstancias particulares actuales.

Este plan está en línea con el Plan de Recuperación, Transformación y Resiliencia (PRTR). Las actuaciones de impulso a la internacionalización de la economía española se verán reforzadas con fondos europeos procedentes del Mecanismo de Recuperación y Resiliencia, instrumentados en España a través del mencionado PRTR.

Este plan traza la hoja de ruta para la modernización de la economía española, la recuperación del crecimiento económico y la creación de empleo, para la reconstrucción económica sólida, inclusiva y resiliente tras la crisis de la COVID-19, y se sustenta en cuatro pilares que vertebrarán la transformación del conjunto de la economía: transición ecológica, transformación digital, igualdad de género y cohesión social y territorial. El plan se estructura en torno a diez políticas tractoras, que van a incidir directamente en aquellos sectores productivos con mayor capacidad de transformación de nuestro tejido económico y social. En concreto, los recursos procedentes del PRTR destinados a la internacionalización se enmarcan en el «Componente 13 de impulso a la pyme», dentro de la Política Palanca V: «Modernización y digitalización del tejido industrial y de la pyme, recuperación del turismo e impulso a una España Nación Emprendedora», y ascienden a 201,9 millones de euros para el periodo 2021-2023.
Se podría concluir, por tanto, que el Plan de Acción 2021-2022 constituye una herramienta de gran relevancia para la economía de nuestro país, al configurarse como una de las principales medidas de impulso y coordinación de la política de internacionalización, debiendo ser clave para reforzar la economía española en la era pospandemia.

\section{Bibliografía}

Ministerio de Economía, Industria y Competitividad (2017). La Estrategia de Internacionalización de la Economía Española 2017-2027. https://comercio.gob.es/es-es/estrategia_internacionalizacion/Paginas/Estrategia-Internacionalizacion-2017-2027.aspx

Ministerio de Industria, Comercio y Turismo (2019). El Plan de Acción para la Internacionalización de la Economía Española 2019-2020. https://comercio.gob.es/es-es/estrategia_internacionalizacion/Paginas/Plan-Accion-Internacionalizacion-2019-20.aspx

Ministerio de Industria, Comercio y Turismo (2019). La Estrategia PASE (Países con Actuación Sectorial Estratégica). https://comercio.gob.es/eses/estrategia_internacionalizacion/Paginas/ Pase.aspx

Ministerio de Industria, Comercio y Turismo (2021). El Plan de Acción para la Internacionalización de la Economía Española 2021-2022. https://comercio.gob.es/es-es/estrategia_internacionalizacion/Paginas/plan-accion-2021-2022.aspx

Ministerio de Industria, Comercio y Turismo (2021). Boletín de Inversiones Exteriores. https://comercio.gob.es/InversionesExteriores/Publicaciones/ Paginas/default.aspx

Subdirección General de Estudios y Evaluación de Instrumentos de Política Comercial (2021). El sector exterior ante 2021: retos y oportunidades. Boletín Económico de ICE (3134). https:// doi.org/10.32796/bice.2021.3134.7184 